\title{
Percepción de los docentes sobre sus acciones innovadoras ${ }^{1}$
}

\section{Diego Ernesto Leal-Fonseca}

https://orcid.org/0ooo-0oo2-6154-6766 Universidad EAFIT, Colombia

dlealfon@eafit.edu.co

\section{Tatiana Ortiz-Pradilla}

https://orcid.org/0000-0001-9947-027X Universidad EAFIT, Colombia tortizpr@eafit.edu.co

\section{Laura Isabel Rojas De Francisco}

https://orcid.org/0000-0002-7250-5599 Universidad EAFIT, Colombia lrojas3@eafit.edu.co

\section{Juan Carlos Monroy-Osorio}

https://orcid.org/0000-0002-0518-5011 Universidad EAFIT, Colombia jmonroyo@eafit.edu.co

\section{Resumen}

El estudio presenta una exploración acerca de las percepciones de docentes de instituciones oficiales sobre sus acciones innovadoras, en Bogotá, Colombia. Se utilizaron métodos mixtos, para explorar percepciones sobre la generación y desarrollo de acciones de innovación educativa, identificar los mecanismos utilizados para difundirlas y conocer las problemáticas asociadas al logro de las actividades innovadoras en la educación pública. Los resultados ofrecen las bases para construir estrategias de difusión y reconocimiento de las acciones innovadoras en la práctica docente, y muestran la necesidad de comprender mejor las condiciones, situación, intereses y posibilidades de los docentes de instituciones oficiales.

\section{Palabras clave (Fuente: tesauro de la Unesco)}

Bogotá, Colombia; educación escolar; estrategia de enseñanza; formación de docentes; formación de profesores; innovación educativa.

1 Investigación resultado del convenio FT-IDP-04-09 mediante contrato No. 095 de 2017 entre la Universidad EAFIT y la Secretaría de Educación y el IDEP

Recepción: 01/04/2019 | Envío a pares: 23/10/2019 | Aceptación por pares: 07/04/2020 | Aprobación: 19/06/2020 


\title{
Teachers' Perception of their Innovative Actions
}

\begin{abstract}
This paper presents an exploration of the teacher's perceptions about their innovative actions, in official institutions of Bogotá, Colombia. Mixed methods were used to explore perceptions regarding the generation and development of educational innovative actions, to identify the mechanisms used to disseminate those actions, and to learn about the problems associated with the achievement of innovative activities in public education. The results offer the grounds to build strategies for the dissemination and recognition of innovative actions in teaching practice and show the need to understand the conditions, situation, interests, and possibilities of those teachers.
\end{abstract}

\section{Keywords (Source: Unesco Thesaurus)}

Bogota, Colombia; schoolchildren education; teaching methods; teacher training; teacher education; educational innovations. 


\section{Percepção dos docentes sobre suas ações inovadoras}

\section{Resumo}

O estudo apresenta uma pesquisa exploratória sobre as percepções que os professores de escolas públicas em Bogotá (Colômbia) têm sobre suas ações inovadoras. Métodos mistos foram utilizados para pesquisar percepções sobre a geração e o desenvolvimento de ações de inovação educacional, identificar os mecanismos utilizados para disseminá-las e conhecer os problemas associados à realização de atividades inovadoras na educação pública. Os resultados oferecem a base para a construção de estratégias de divulgação e reconhecimento de ações inovadoras na prática docente, e mostram a necessidade de aprofundar e compreender melhor as condições, situações, interesses e possibilidades dos professores en relação com os processos de inovação.

\section{Palavras-chave (Fonte: tesauro da Unesco)}

Bogotá, Colômbia; estratégia de ensino; educação escolar; treinamento de professor; Inovação educacional. 
Como parte del Plan de Desarrollo Bogotá Mejor para Todos 2016-2020, la Secretaría de Educación de Bogotá adelantó el proyecto de inversión 1040, denominado Bogotá reconoce a sus maestros, maestras y directivos como líderes de la transformación educativa (Secretaría de Educación de Bogotá, 2016). El proyecto nace de una necesidad puntual planteada por el Instituto para la Investigación Educativa y el Desarrollo Pedagógico (IDEP), quien puso en marcha un proceso de formulación de una estrategia para la divulgación y la comunicación de las acciones innovadoras de docentes de la ciudad de Bogotá, el cual fue desarrollado por la Línea de Investigación y Desarrollo en Informática Educativa de la Universidad Eafit, con la participación de profesores del departamento de mercadeo de esta misma institución

En este proceso, se buscó entender el contexto de la ciudad desde la perspectiva de la política pública en materia de innovación educativa, para identificar a los actores y acciones clave que hacen parte del escenario sobre el que se pueden desarrollar iniciativas a futuro. Igualmente, se exploró un conjunto de elementos conceptuales que permitieron delinear el marco de análisis para la difusión de la innovación educativa, el cual sirvió como insumo para la realización de un estudio exploratorio de actividades que los docentes de las instituciones educativas oficiales de la ciudad de Bogotá llevan a cabo para difundir sus acciones innovadoras.

El estudio utilizó métodos mixtos para explorar percepciones sobre aspectos relacionados con la generación y desarrollo de acciones de innovación educativa, así como sobre los mecanismos utilizados para difundirlas y las problemáticas asociadas. Dicha investigación no se había realizado antes en la ciudad, lo que permitió vislumbrar parte del ecosistema de innovación educativo local y posibilitar -más adelante- el diseño de estrategias que propiciaran en los docentes más acciones innovadoras y su difusión. En este sentido, además del objetivo mencionado en el párrafo anterior, se buscó explorar percepciones sobre las acciones de innovación educativa, identificar los mecanismos de difusión en curso y conocer las problemáticas a las que se enfrentan las actividades.

Este documento se enfoca solamente en la presentación de los resultados obtenidos en dicho estudio, los cuales sirvieron como insumo para la formulación de un conjunto de recomendaciones encaminadas a mejorar los flujos de información que hacen parte del ecosistema de innovación educativa de la ciudad. En ese sentido, este artículo se hace con el objeto de exponer el ecosistema que se da en el quehacer educativo oficial y permitir que las acciones innovadoras de los educadores encontradas en el estudio trasciendan el contexto de la ciudad de Bogotá y permitan que más educadores puedan hacer uso de estas y pensar cómo difundir sus acciones. Además, es un reconocimiento a las labores docentes que apuestan por la innovación.

Este artículo se estructura en torno a un marco conceptual que sustenta las decisiones preliminares de la metodología, una descripción del proceso metodológico, la presentación de resultados destacados y la exposición del logro de objetivos, las limitaciones y las oportunidades de estudio.

\section{Marco teórico}

Para abordar los procesos de divulgación y comunicación de las acciones innovadoras que realizan los docentes de las instituciones educativas oficiales de Bogotá se propuso un acercamiento desde la perspectiva de la difusión de la innovación educativa, con un enfoque que entiende a la ciudad como un ecosistema de innovación educativa. Con este acercamiento se busca contribuir con nuevos elementos a la comprensión del concepto innovación educativa.

La innovación educativa es un concepto amplio, con una historia que abarca varias décadas -desde el trabajo seminal de Huberman (1973)- y comprende diversas definiciones, que pasan por las formas de las innovaciones educativas, los procesos 
vinculados a su desarrollo y los fines que persiguen. Se trata de un concepto multidimensional y polisémico, que debe refinarse y compartirse socialmente, y que es más complejo que sus descripciones o modelos, en la línea de la perspectiva que propone el Programa de las Naciones Unidas para el Desarrollo, al referirse a conceptos similares como el de calidad educativa (PNUD, 2015).

En este sentido, se acerca a una "idea-fuerza" que puede movilizar la acción individual, social y colectiva. Por ello, no puede hablarse de una mejor o peor definición o postura sobre innovación educativa. Por el contrario, las miradas e imaginarios de diversos actores requieren ser reconocidos y armonizados para avanzar hacia acuerdos colectivos que orienten la acción individual.

Teniendo en cuenta lo anterior, en la literatura sobre el tema es posible identificar una serie de elementos comunes que sirven como punto de partida. En primer lugar, una innovación es diferente al simple cambio, pues en su origen se encuentra la intención deliberada y planificada de solucionar un problema o mejorar una situación existente, generando un valor nuevo (Barraza, 2005; Huberman, 1973; Unesco, 2016).

Sin embargo, lo nuevo no se entiende necesariamente como algo nunca antes inventado, sino que admite también algo que ya ha sido conocido o utilizado en otros tiempos o situaciones, pero que ahora se utiliza en nuevas circunstancias, con diferentes finalidades, en diversas combinaciones o formas de organización. El contexto juega un papel primordial en la percepción de qué se denomina como innovador, pues lo que puede ser innovador en un contexto puede no serlo en otro (Barraza, 2005; Cortés, 2002; Tobin, 2010, p. 52).

En esta línea, la introducción de elementos novedosos en sí misma no representa innovación. Las innovaciones educativas se configuran a través de unos contenidos y de una imagen definida de la escuela deseada, apuntando a la transformación y mejoramiento de recursos, metodologías, procedimientos, estructuras, saberes escolares específicos, comunidades y contextos (Garrido et al., 2011; Hazen et al., 2012, p. 303). Por esta razón, se encuentran condicionadas y son influidas directamente por las dinámicas propias del contexto en el que se presentan (Barrantes, 2001).

En una perspectiva más amplia y citando a Cortés Salcedo (2016), el IDEP plantea que "innovar significa abrir horizontes, generar un interés investigativo, disfrutar el placer de indagar, descubrir proponer, revaluar, pero ante todo de inventar. Se trata de avanzar en una postura crítica frente a los postulados existentes, pues sólo cuando se convierte en tema de reflexión, de investigación, de cuestionamiento, es posible la innovación" (2016, p. 3). En esta vía, y citando a Gómez (2016), el IDEP señala que "a pesar de lo etéreo del término, la innovación pedagógica alude a la sistematización y al reconocimiento de prácticas transformadoras y como tal es 'una oportunidad para visibilizar y reconocer las prácticas pedagógicas en función de las necesidades del contexto'" (p. 3).

Para efectos de este estudio, es de interés complementar la comprensión de la innovación educativa con un abordaje del contexto en el cual ocurre. Desde una perspectiva ecológica comprendemos el contexto como un ecosistema: el resultado de la suma de las respuestas individuales a estímulos recibidos de elementos ambientales, donde un complejo conjunto de relaciones y condiciones viven en equilibrio, con procesos que se relacionan entre sí y desarrollan en función de factores físicos de un determinado ambiente (Álvarez et al., 2011; Jackson, 2012).

Esta mirada ha encontrado resonancia en el sector productivo en la última década. En un ecosistema de innovación, diversas dinámicas y asociaciones suceden entre individuos, comunidades e instituciones, con el objetivo de generar y desarrollar nuevas visiones y propuestas que posibiliten el progreso en 
múltiples campos, en beneficio de la sociedad (Carlson y Wilmot, 2006). Se trata de redes complejas y cooperativas entre los interesados en una industria particular, cuyos esfuerzos de colaboración generan nuevas ideas, productos y procesos para los consumidores de la industria (Abdul-Jabbar y Kurshan, 2015). Así, un ecosistema de innovación no es estático; por el contrario, las cambiantes relaciones entre sus actores le otorgan un dinamismo en el tiempo que conduce al cambio y la evolución. Debido a su complejidad, un ecosistema de innovación puede ser influenciado y encauzado, pero no necesariamente controlado, por lo que se requiere de una continua revisión de su carácter, situación y potenciales.

Desde la perspectiva de Choque-Larrauri (2009), un ecosistema de innovación educativa puede analizarse en múltiples niveles. En el nivel micro está la escuela, en la cual interactúan los estudiantes, los profesores, los materiales educativos, el espacio físico y social donde se realizan las actividades educativas, los espacios deportivos y de recreación, los servicios básicos, las normas, reglas, etc., incorporando población, medio ambiente, tecnología y relaciones organizadas; en el nivel meso se presentan otros entornos inmediatos donde las personas interactúan, como son la familia, el servicio de salud, el municipio o cualquier espacio donde interactúan permanentemente las personas; el nivel exo es el de influencia de la política educativa local, el trabajo de las familias, la organización de la comunidad y en especial los medios de comunicación, donde también circula el saber; y en el nivel macro se tienen factores tecnológicos y de comunicación, factores filosóficos, religiosos y éticos, factores psicológicos, sociales y familiares, factores culturales y estilos de vida, el sistema educativo, factores políticos, administrativos y legales, económicos y laborales, y biológicos y de salud.

Un ecosistema de innovación educativa depende entonces de la existencia de interconexiones sociales, participación conjunta y comunicación fluida (Álvarez et al., 2011), orientados a producir condi- ciones que contribuyan a la generación y difusión de innovaciones educativas. Así, la perspectiva ecosistémica invita a considerar los procesos que dan origen a las innovaciones educativas, ampliando el espectro para referirse no solo a la innovación educativa, sino a las acciones innovadoras que la hacen posible. En este sentido, Markmann (2012) señala que entender cómo desarrollar buenas ideas y cómo transformarlas en planes factibles es tan importante como el desarrollo de tales planes.

Como consecuencia de esto, los procesos de comunicación y divulgación de las acciones innovadoras -no solo de las innovaciones como producto terminado- adquieren una gran relevancia y, en procesos de difusión de acciones innovadoras, responden al proceso de reconocimiento que se viene dando en el contexto de la educación del distrito (IDEP, 2019). No obstante, el desafío no es solo divulgar, sino conseguir el movimiento y adopción de la innovación por parte de nuevos actores de un sistema social. Esto es, se trata de un proceso de difusión de la innovación en el que los grupos objetivo conozcan, adopten y utilicen rutinariamente innovaciones educativas (Ahmed et al., 2011; Hazen et al., 2012; Lomas, 1993; Rogers, 2003).

Para pensar en difusión es fundamental considerar las particularidades no solo de una innovación, sino del adoptante' y su entorno (Melville y Ramirez, 2008; Zhao et al., 2002). Para difundir y comunicar efectivamente las innovaciones educativas, es crucial entender de la manera más precisa posible las necesidades, deseos, prioridades, hábitos y capacidades de sus posibles adoptantes. No solo porque cada innovación posee un conjunto de características específicas -las cuales pueden proporcionar una base para explicar las diferencias que facilitan su adop-

1 Conviene señalar aquí que al hablar de adoptantes no se presume exclusivamente que una innovación educativa se adopta en el sentido de ser replicada. Al hablar de adoptante, se hace referencia al individuo que encuentra inspiración en una innovación existente para avanzar en la generación de innovaciones propias -las cuales pueden o no ser una réplica de la innovación original. 
ción-, sino porque el conocimiento y las competencias para innovar dependen del contexto y del tipo y la clase de contribución que aportan a la educación (Shapiro et al., 2007).

\section{Objetivo del estudio}

Identificar las percepciones de los docentes de instituciones educativas de la ciudad de Bogotá en relación con la difusión de sus acciones innovadoras.

\section{Metodología/método}

$\mathrm{El}$ artículo se basa en un estudio exploratorio con la aplicación de métodos mixtos de recolección de la información y con propósitos de validación y contrastación. El estudio se enmarca en el paradigma interpretativo/constructivista, que afirma que los seres humanos construyen significados a medida que interactúan y realizan sus actividades e interpretan contextos, objetos y a otros individuos, de acuerdo con su significado para el individuo, y al hacerlo dan sentido a la actividad que están haciendo (Schwandt, 1994). El estudio a su vez recurre a categorías de análisis fundamentadas en la teoría ligada a la definición de ecosistema para la comprensión de los procesos relacionados con la innovación y la educación conjuntamente, para lo cual también se recurre a referentes de estudio sobre ecosistemas de innovación educativa (Abdul-Jabbar y Kurshan, 2015; Hazen et al., 2012; Shapiro et al., 2007).

Por la aplicación de distintos métodos de recolección, el estudio se desarrolló en dos etapas paralelas en relación con la recolección: la primera, de orden cuantitativo, se valió de encuestas realizadas de manera continua durante el desarrollo del proyecto, con el propósito de identificar perfiles de participación y validar aspectos que fuesen surgiendo de la participación; la segunda, de naturaleza cualitativa y en sesiones de grupo, se enfocó en explorar percepciones sobre la generación y desarrollo de acciones de innovación educativa, identificar mecanismos utilizados para difundirlas y conocer las proble- máticas para lograr las actividades innovadoras en la educación pública.

La primera etapa recurre a una encuesta cuyo propósito es identificar los aspectos más significativos de la noción de acciones innovadoras. Con este fin, la formulación del instrumento se vale de definiciones que se sustraen de la teoría consultada, para con ello corroborar las variables a tener en cuenta en una estrategia de difusión de la innovación educativa y hacerlo teniendo en cuenta el contexto de la ciudad de Bogotá. Además, sirvió como mecanismo de validación de algunos aspectos identificados en el marco teórico y situacional en relación con las nociones de innovación educativa y los componentes del ecosistema distrital de innovación educativa.

Para la recolección de la información, se elaboró un instrumento que contempló las siguientes temáticas: información demográfica, espacios de socialización, concepciones sobre innovación educativa, generación de la innovación, apoyo a la innovación educativa y divulgación de acciones de innovación educativa. La encuesta incluyó preguntas cerradas con respuestas de única opción y opción múltiple y preguntas mixtas -preguntas cerradas donde una de las opciones es una pregunta abierta de respuesta libre-. El instrumento se diseñó de esta manera para validar aspectos generalizables, verificar variaciones en las variables y facilitar que emergiesen nuevos aspectos.

La población objeto de la encuesta correspondió a docentes y directivos docentes de la ciudad de Bogotá, que respondieron a una convocatoria abierta en la que se buscaba que participaran de manera voluntaria y que ejercieran actividades en instituciones oficiales de educación pre-escolar, básica y media con diferentes áreas de formación. Quienes participaron declararon diferentes niveles de educación en licenciatura o pregrado, especialización, maestría o doctorado.

El tamaño de la muestra fue de 387 personas y se estableció como tope de cierre usando como refe- 
rencia una población finita cualitativa del universo constituido por 36.000 docentes y directivos docentes. Se utilizó un cálculo muestral con un nivel de confianza de $95 \%$, error máximo de $1 \%$ y desviación estándar de 3,87, correspondiente a una muestra mínima de 58 individuos.

El instrumento se aplicó a través del software Qualtrics a un grupo aleatorio de docentes y directivos docentes que respondieron a la convocatoria de manera virtual, mediante envío por las bases de datos del IDEP y la Secretaría de Educación Distrital, y se distribuyó a redes de innovación docente, movilizado a través de redes sociales con tres influenciadores en el tema de innovación educativa. Igualmente, se compartió con los participantes asistentes al Foro Educativo Distrital a quienes se aplicó de manera administrada y se utilizó en las primeras sesiones de los talleres con aquellas personas que no hubiesen participado en esta recolección de información.

El perfil de las personas participantes estuvo compuesto por 55 directivos docentes y 332 docentes, que ejercen sus actividades en educación media $(24 \%)$, secundaria (32\%), primaria (31\%) y preescolar (12\%); con niveles de educación en licenciatura o pregrado (25\%), especialización (20\%), maestría (50\%) y doctorado (5\%).

Por razones de alcance y por la posibilidad de dar voluntariamente información, la encuesta se centró en información sobre aspectos puntuales de la situación de los docentes y directivos docentes en relación con la aplicación o conocimiento de innovación educativa. No obstante, este ejercicio inicial sirve como insumo para estudios futuros y de mayor alcance que vienen siendo realizados por la Secretaría de Educación Distrital.

Una segunda etapa comenzó una vez se logró una base importante de participantes en sesiones de grupo, cuyo propósito fue identificar acciones de comunicación y difusión de acciones innovadoras de los docentes y directivos docentes de instituciones educativas de Bogotá, en un espacio que se estructura y ejecuta con base en metodologías de cocreación, cuyo diseño se apoyó en los elementos conceptuales surgidos del marco situacional y teórico, de manera que los docentes compartieran sus propias experiencias de innovación educativa. Para validar el instrumento, se hizo una revisión con expertos en metodologías de cocreación y con el IDEP. Así mismo, en el primer taller se corroboró que los resultados obtenidos correspondieran con lo buscado en los objetivos, además de exponer aspectos adicionales.

Las sesiones contaron con la participación de docentes ganadores del Premio de Investigación e Innovación Educativa del Distrito, así como de líderes de redes de docentes de la ciudad, quienes sumaron un total de 100 personas distribuidas en ocho talleres. Cada sesión estuvo compuesta por dos momentos. El primero se encaminó a identificar, a partir de la experiencia de los participantes, los aspectos de una comunicación efectiva que deben ser tenidos en cuenta en la formulación de una estrategia de comunicación. También sirvieron como excusa para compartir experiencias y como base para fundamentar el valor añadido de la innovación a partir del valor único que cada individuo genera en su propia experiencia -considerada de primera generación-, la que, al ser compartida con otros, se convierte en experiencias de segunda generación basadas en un proceso de cocreación, donde se comparten y relacionan valores personales, sociales y culturales (Prahalad y Ramaswamy, 2003). El segundo momento contribuyó a la identificación colectiva de acciones de comunicación y difusión para las acciones innovadoras de los docentes del distrito.

Las discusiones plenarias fueron grabadas y transcritas, para ser trabajadas mediante análisis temático (Aronson, 1994), para encontrar las categorías de análisis que se desarrollan en los hallazgos de este documento y que se componen de: 1) acciones de innovación educativa, 2) componentes de la estructura para la difusión, 3) soporte para el aprovechamiento, promoción y participación de las 
acciones, y 4) su rol en el ecosistema. Cuando las categorías compartidas comenzaron a coincidir en las mismas temáticas, se realizó el análisis mediante descripción densa (Geertz, 2003) y la posterior triangulación de información proporcionada por la revisión documental, los datos de la encuesta y las evidencias de las narraciones de los talleres.

El resultado de los talleres fue articulado en un esquema estratégico para la divulgación de acciones innovadoras, que no hace parte de este documento. Es de tener en cuenta además que el tiempo de ejecución del proyecto impidió hacer un análisis de relaciones semánticas que orientaran la construcción de categorías de discusión y evaluación. Sin embargo, se desarrolló un documento donde se extraen las categorías directamente de las transcripciones de cada taller realizado. Todos los insumos fueron entregados al IDEP para apoyar nuevas investigaciones en esta área.

\section{Hallazgos}

Los participantes en el estudio señalaron que la innovación educativa sucede cuando en el aula se puede "poner un problema y ver qué se construye de este, dando pauta a la creatividad y la innovación de manera que no se pierden las ideas en un sistema creado para repetición y obediencia". Además, consideran que la motivación para innovar depende de ambientes que no sean hostiles a su desarrollo, donde se puedan realizar acciones, con tiempo suficiente y recursos, para que luego se hagan visibles y tengan reconocimiento positivo de los pares. Para llegar a esto, se requiere poner en conocimiento de toda la comunidad aquello que se pueda apreciar como innovación. Este estudio muestra cómo la población participante desarrolla estos procesos, qué se puede hacer y cuáles son los retos y dificultades que enfrentan.

\section{Generación y desarrollo de acciones de innovación educativa}

Los participantes reconocen que quienes hacen innovación educativa en Bogotá son las redes de docentes, las universidades y los docentes, pero consideran a los estudiantes como actores clave, porque son la inspiración. Destacan la labor del IDEP - por su papel en el apoyo y desarrollo de la innovación educativa- y de Colciencias -por su rol en relación con la investigación-. Además, reconocen como actores relevantes en el apoyo a la generación de la innovación educativa a nivel local, en orden de importancia, al Ministerio de Tecnologías de la Información y las Comunicaciones (MinTIC), al Instituto Distrital de las Artes (Idartes), a la Secretaría de Educación Distrital, al Centro de Innovación Educativa Regional, al Ministerio de Educación, a la Fundación Compartir, a las direcciones locales de educación y a la Fundación Dividendo por Colombia.

Entre los principales comentarios se señala que "todos somos innovadores, todos tenemos acciones para contar, y conocer a quien hace cosas que pueden ayudar a movilizar". En este sentido, la innovación en la educación se entiende como "un proceso pensado y planificado para transformar una realidad problemática y no es solo aplicar algo diferente o llevar otra clase de recursos". En el contexto educativo "se aplica a problemas reales", por eso conlleva "reflexión y pensamiento crítico", que requiere tanto de las acciones en sí mismas como de la sistematización de experiencias.

Aunque puede ser un proceso planificado o provenir de cualquier cambio, las acciones de innovación educativa se inspiran en "las necesidades de los estudiantes, las prácticas pedagógicas y por el deseo de estar al día y avanzar al ritmo de sus estudiantes". Requieren contar con recursos y espacios para la experimentación, de formación en innovación educativa, del trabajo en red o del acompañamiento.

\section{Canales de apropiación utilizados}

Los participantes señalan que sus fuentes en temas de innovación corresponden a publicaciones como Aula Urbana, Magisterio y Palabra Maestra de la Fundación Compartir. También páginas de la Secretaría de Educación, del Ministerio de Educación 
o repositorios de universidades. Son considerados en menor medida los eventos culturales y los espacios digitales disponibles en línea, como blogs, redes sociales, correos y WhatsApp. Los procesos y herramientas institucionales, como informes de gestión, páginas web institucionales, boletines institucionales, redes sociales institucionales y encuentros directivos, se usan, pero su valoración no es alta en relación con su pertinencia para la difusión.

También reciben información "a través de charlas pedagógicas entre pares, de foros, jornadas pedagógicas, redes sociales como Facebook, nodos de investigación, redes académicas, el correo electrónico". Se mencionan también el "canal de YouTube de la Red de Investigadores o Sala de Profes, donde publican se generan contenidos sobre casos de éxito, herramientas de aula, tareas semanales y se ampliar la red al conectarse con otros". Las publicaciones propias de los docentes son otro ejemplo, y hay quienes "escriben cuentos, poesía, que están en contacto con editoriales o tienen la posibilidad de viajar a distintos lugares y cuentan sus historias" en blogs, páginas de redes sociales o páginas web propias.

La forma en que llega la información a cada uno puede ser diversa. Explican que, por un lado, "hay grupos informales y profesionales de WhatsApp, páginas de Facebook" o "canales más formales relacionados con las jornadas pedagógicas, que dan a conocer experiencias que han dado resultado" y sirven al propósito de "inspirar al compartir la acción innovadora", y se "reconocen las experiencias exitosas". Finalmente, refuerzan que todo puede estar articulado con "reuniones de docentes" y sistematizado en vídeos de creación y boletines institucionales.

Al referirse a los canales, admiten que es necesario "no confiar solamente en lo institucional", sino pensar en "canales para publicación propia, como redes sociales, páginas de Facebook”. Aunque pueden ser muy diversos, a veces no se usan "los canales de comunicación a nivel de las instituciones educati- vas" y esas "fuentes de inspiración que son referente, como la publicación académica, las bases de datos de universidades reconocidas, hoy en día se van mezclando" con "las redes sociales, los servicios de noticias, Google". Entonces, para "saber qué está pasando, inspirarse y generar ideas", estas se deben incluir.

\section{Mecanismos para la divulgación de las acciones de innovación educativa}

Se evidencian como esenciales los aspectos de relacionamiento y socialización que llevan al trabajo en equipo, donde el compromiso, la buena comunicación, las relaciones e interacción entre todos los integrantes de la comunidad y otros participantes son, en conjunto, factores determinantes para contribuir con una cultura de colaboración.

Por eso, las acciones innovadoras que se realizan se dan a conocer en primer lugar mediante la conversación informal con compañeros, en lugares cotidianos como la cafetería, durante descansos en el colegio, en reuniones y actividades sociales con profesores, compañeros de estudios, colegas o con quienes se encuentran al asistir a eventos académicos. No obstante, al mismo tiempo se señala la necesidad de contar con más espacios de socialización informal.

Entre los eventos se mencionan foros, encuentros, concursos, coloquios y se destacan los realizados por la Red Distrital de Docentes Investigadores, el Foro Educativo Distrital, la mesa local de coordinadores o los encuentros con estudiantes y padres. Se evidencia que los mecanismos más relevantes para la difusión son los eventos y las publicaciones académicas, entre los que destacan aquellos gestionados por el IDEP.

Por su parte, las iniciativas mejor valoradas en su aporte a la difusión de acciones innovadoras son el Portal Educativo Colombia Aprende, Red Académica - portal de la Secretaría de Educación Distrital-, el Centro de Documentación del IDEP, el Foro Educativo Distrital y el portal institucional de la Secretaría de 
Educación de Bogotá. En menor medida, mencionan la Red Distrital de Bibliotecas Públicas, el Foro Educativo Nacional y los Puntos Vive Digital, y agregan la Feria de Experiencias de Ondas. El Centro Móvil de Innovación y el ViveLab Bogotá, por su parte, son escasamente reconocidos, lo cual puede explicarse por su reciente aparición en el entorno distrital al momento de la realización del estudio.

\section{Sistematizar y compartir la experiencia}

Recolectar la información de proyectos que han llevado a soluciones o han sido identificados como casos de éxito puede demostrar los alcances de lo que se hace, confrontar la crítica, aportar al área y compartirse y así motivar a otros. Aunque algunos participantes exponen barreras relacionadas con la "falta de habilidades o desmotivación para escribir", es posible hacerlo con lo que hacen muy bien: "contarlo, recurrir a lo oral", "porque la palabra es una herramienta de los maestros que conecta, invita, incita, motiva, inspira, engancha".

Pero pueden encontrarse con problemas para documentar y sistematizar, por no tener espacios para hacerlo o no contar con una comunicación oportuna que les permita prepararse y darse a conocer en eventos como el Premio a la Investigación y a la Innovación del IDEP o aprovechar las oportunidades que aparecen en las páginas de la Secretaría.

Esto reafirma la necesidad de reconocer qué actividades para la difusión de acciones innovadoras se llevan a cabo y son efectivas para difundir lo que se hace en innovación educativa en la ciudad, porque consideran que "no existen canales internos de comunicación que contacten a los profesores innovadores o que visibilicen las innovaciones", o porque "no hay escenarios, o espacios constantes donde se pueden conectar redes, experiencias, personas o docentes de los sectores privado y público".

Los participantes proponen la puesta en marcha de mecanismos para clasificar a quiénes dirigir esa información, plantear temas y reforzar eventos "en que se han mostrado realidades y situaciones" de manera que "toda la gente conozca lo que se hace, las variadas problemáticas de los colegios y cómo se ha pensado en transformarlas". En cuanto a las publicaciones, proponen que "el IDEP en vez de publicar un libro con contenidos de 15 páginas recoja la investigación seleccionada en series de libros" y crear o fortalecer con el uso "bases de datos donde se puedan encontrar todos los proyectos por temas", así como un "repositorio digital, con un mapa de experiencias que permita búsquedas refinadas por tema".

Si bien reconocen que hay que publicar aprovechando las convocatorias del IDEP o de revistas indexadas o no indexadas, los participantes señalan que se deberían considerar medios electrónicos, que permiten acceder a más gente y exponer artículos sin arbitraje y pueden ser tan o más efectivos para llegar a quien interesa. Para eso además plantean "acompañamiento en la escritura, para todos, no solo con quien se postule, y contar con talleres", pues motiva saber "que todos pueden ingresar y participar".

También se puede aprovechar la existencia de periódicos en los colegios. Mencionan "Bio- Parceros, una página alimentada con los mejores trabajos, con producciones de estudiantes, donde se integran lecturas, agregan enlaces o videos" y proponen que "dentro de la página web de la Secretaría haya espacio para que cada Institución desarrolle allí su página web y publiquen lo que van haciendo por áreas o localidades". Con eso consideran que se "genera diálogo interdisciplinario y se recupera la cultura escrita" y se logra que "todos tengan las mismas oportunidades, romper círculos viciosos, tener reemplazos para facilitar la jornada para que los docentes escriban sin dejar la responsabilidad educativa".

\section{Aprovechar el valor de las acciones innovadoras en educación}

Uno de los comentarios recurrentes es que las ideas y los conceptos llevan a la indagación, a escudriñar, a tener pretextos y a crear prácticas y proyectos para encontrar una solución, y dan un ejemplo: 
con la pregunta "¿por qué cuando llueve las cosas se ensucian más?, se generó un ejercicio en clase que llegó a temas como filtros en botellas, el ruido escolar y dispositivos para medirlo, rescate cultural y finalmente a un proyecto de apropiación de la música y danza como herencia africana en Colombia con la realización de un musical". Así, la innovación surge, se cuenta, se vivencia.

La inspiración, aunada a la contextualización y la reflexión, conlleva prácticas que derivan eventualmente en proyectos que adquieren sentido. Especialmente "ahora con tanta tecnología para buscar conocimientos, se necesita enseñar y aprender a pensar"; se trata de "trabajar sobre herramientas de adaptación al cambio, de acuerdo a la situación; desaprender cosas y no solo repetir" en un "aprendizaje vivencial". Así, con la "participación de estudiantes" pueden expandirse los proyectos orientados a la innovación. Conocer "las cosas que se emplean en el aula, que sirven como referente para ver que sí es posible con esos recursos que tiene la institución", inspira tanto al "grupo que siempre le gusta estar innovando, como al grupo que se resiste o sabotea".

Luego es preciso tener en cuenta que los avances conseguidos pueden diluirse si, "estando la misma comunidad, los mismos docentes y proyectos, cambian las políticas, cambian las administraciones y cambia todo". Por eso "los docentes deben liderar las innovaciones y no esperar que vengan las entidades a decir qué hacer, tienen que conectarse para avanzar y apoyarse entre entidades", porque "la pedagogía se hace desde la acción, no la crítica”, por "los mismos docentes que están en red, que quieren ir a las Instituciones y hablar con pares" que lo ven como un reto para "motivar al otro a investigar y hacerlo en llave, en red".

Hay procesos de gestión que deben ser revisados con respecto a exigencias y al apoyo para lograrlas, y se ha de considerar el alcance y las posibilidades, de manera que no se dé la fragmentación o postergación de actividades, se pierda el compromiso o haya temor a mostrar lo que se hace. Expresan que para llegar a los docentes hay que "seducir con el ejemplo, no imponer". Los mismos docentes son quienes incentivan "a sus compañeros, para que se reconozcan las experiencias positivas" y en el proceso se integra a los estudiantes con quienes se desarrollan las experiencias pedagógicas.

Es evidente que el reconocimiento es un factor que resulta esencial, por lo que no puede ser eventual, sino que tiene que darse de forma continua a partir de la labor cotidiana. Es en este sentido que la difusión se torna fundamental, al contribuir de manera directa al reconocimiento de la labor docente.

\section{Agendar la participación y promover los encuentros}

Los participantes exponen que es difícil compartir espacios, porque "se tiene que cumplir con lo académico y socializar con todos es complejo". Pero proponen "la reunión de área, entre compañeros" y pensar en cómo lograr que "cada docente reconozca su experiencia" frente a los asistentes, "para que todos los demás compañeros la reconozcan y se logre un encadenamiento para trabajar". Así se da ánimo, que se manifiesta en un "entonces qué hacemos para volverlo a hacer".

Dado que el tiempo para abrir espacios para los docentes es escaso, plantean que se hagan semanas institucionales "para desarrollar habilidades en los docentes" o "generar centros de interés", con iniciativas como "un comité de innovación o un colectivo de innovación", o que estén en la misma institución en espacios para socializar experiencias e identificar que hay innovación; y que quienes lo hagan "no sean nombrados a dedo" o tengan que "solicitar permiso en contrajornada". Así se pueden "recoger experiencias innovadoras, escribirlas o socializarlas al resto del colegio, para movilizar".

En cuanto al Foro Educativo Local, se reconoce que "intenta lograr que los profesores puedan socializar parte de lo que se hace", pero entonces se 
requiere de espacios dentro de la Institución "para divulgar" lo que se necesite. Así cuando se realizan los Foros Institucionales, ya se sabe "qué están haciendo en preescolar, primaria, bachillerato, en la jornada de la mañana, la jornada de la tarde, y qué se está trabajando en cada una de las sedes". La motivación comienza en "espacios para visibilizar y divulgar lo que ocurre en las Instituciones", pero que no pase que "en un solo día o en una sola semana haya cinco actividades y tiene que ir un docente de cada área y hay que elegir". También hay que partir de los trabajos que tienen los profesores que son innovadores, dar a conocer esos trabajos y experiencias, en "talleres prácticos donde se vivencie lo que hicieron con sus estudiantes".

\section{Actividades y responsabilidades en el apoyo y difusión de las acciones innovadoras}

Para pensar en una forma de articular esfuerzos, se menciona la investigación como eje, porque "el eco se encuentra generalmente en las Instituciones de Educación Superior" y para esto "se requiere sintonía por parte de coordinadores y directores con los docentes, con respecto a lo que hacen, dicen o están estudiando en sus posgrados". Así, hay idea de cómo podrían "encaminarse otro tipo de currículos en la Institución" y dar a conocer "las variadas problemáticas de los colegios" o servir como insumo para "transformarlas de forma innovadora". Saber quiénes son los docentes, en términos de formación, permite "aprovechar el saber de los docentes y demostrar que no solo en la universidad se produce conocimiento".

Además, es posible que se generen "líneas de investigación como parte de unas políticas" para que avancen las propuestas; eso implica "que sigan cuando cambie la administración". Para lo cual, además, proponen que los directivos, "que son los que pueden convocar", lo hagan apoyados en políticas públicas y así faciliten tiempo para "pensar las cosas más profundo y largo" y permitir a los docentes encontrar cómo "sensibilizar a partir de realidades impactantes, que hacen parte del día a día, y afectan el trabajo pedagógico"; eso es "reconocer el docente como académico".

Las redes son la mejor forma de "poder compartir con otros compañeros del mismo ciclo, con los mismos intereses y problemáticas", aunados a "los diplomados de la Secretaría" que son un eje para "enterarse de muchas cosas y motivar a compañeros que se desaniman, porque el que no hace, desmotiva al que sí". Las redes sirven, "porque allí se congregan intereses comunes". Pero eso implica "solicitar tiempos para estar indagando sobre qué están haciendo en los otros colegios, qué hay de innovación, cómo traer una idea de otro colegio a la institución". Esos espacios de reflexión de la red posibilitan encontrarse "con recursos humanos capacitados, idóneos, con gente que ha profundizado en estos estudios, compañeros con capacidades y mucha experiencia".

Sobre el reconocimiento, también plantean la "bonificación para los docentes que publiquen artículos en revistas especializadas, en revistas académicas", lo cual propicia “diálogo pedagógico, más allá de la conversación de cafetería que, aunque es efectiva, debe llevarse a producir", por lo que sí se piensa en "tener una persona responsable que se asegure que no se quede en el encuentro, que queden memorias". Se trata de transmitir a los compañeros y directivos docentes "que hay canales, hay espacios, hay gente, hay movilidad, que se están haciendo cosas", y para enterarse la mejor manera es hacerlo dentro de la institución y "luego otras instituciones se van uniendo y al final eso termina impactando en compañeros que son reticentes e irradiándose en la ciudad".

\section{Consolidar un modelo local para la difusión de acciones de innovación educativa}

"No hay otra ciudad como Bogotá, ninguna otra ciudad en el país tiene todo el contenido que ha producido el IDEP sobre innovación educativa, pero podemos contar mejor el asunto". Por eso la innovación "debe ser una política" que se articule a nivel 
institucional y "pueda guiar cómo evaluar la producción, la efectividad", planteando rutas claras de mediano y largo plazo.

\section{Conclusiones}

El estudio logra identificar las percepciones de los docentes que participaron sobre sus acciones innovadoras. Esto se logra a partir de los talleres de cocreación, que hace posible identificarlas en el proceso y comprender cómo llevar a cabo acciones de innovación en su labor educativa. Igualmente se logran identificar los aspectos significativos para comprender cómo se dan procesos de innovación en la educación y verificar aspectos relacionados con la forma en que se pueden dar a conocer estas acciones en una estructura, para su difusión, de forma que se reconozcan sus acciones y la importancia de su rol en un conglomerado de procesos que se dan en un contexto y que pueden considerarse como ecosistema.

Pensar el problema de la difusión de la innovación educativa desde una perspectiva ecosistémica hace necesario considerar el papel que desempeñan los diversos actores que componen los diferentes niveles de un ecosistema de innovación educativa. Como se señaló en el marco teórico, no se trata solamente de divulgar -actividad que representa una parte importante de los esfuerzos de las entidades con mayor incidencia en los procesos de difusión de la ciudad-, sino de lograr la movilización de los actores que están en capacidad de generar acciones innovadoras. De ahí la importancia de este primer ejercicio de exploración y de entender cómo un segmento de la población docente de la ciudad aborda los procesos de difusión, comunicación y divulgación, un tema que no había sido explorado a nivel local, donde la comprensión de la innovación en un contexto es de vital importancia.

Por lo anterior, los resultados aportan al tema de la innovación en la educación, al abordar las acciones innovadoras y entender que se gestan en un ecosistema. Se expone un aspecto relacionado con el contexto, en el que se pueden ver las diferentes dimensiones de la innovación educativa enunciadas en su conceptualización (Huberman, 1973), y así se expone que la innovación comporta un proceso de cambio sistémico en el que se da solución a diversos problemas y con ello se mejora el sistema (Barraza, 2005), y como las dinámicas del contexto influyen (Barrantes, 2001), verlas en un ecosistema permite reconocer la complejidad de esfuerzos, colaboraciones, procesos, desarrollo de ideas, productos ( AbdulJabbar y Kurshan, 2015; Jackson, 2012).

Si bien este estudio tiene unas claras limitaciones en términos de alcance, los resultados resaltan la importancia de seguir dimensionando y comprendiendo lo que significa la intención de la actual Secretaría de Educación de reconocer a las maestras, maestros y directivos docentes del Distrito (Alcaldía de Bogotá, 2019; IDEP, 2016; Secretaría de Educación de Bogotá, 2019). Reconocer no se trata solamente de premiar, sino de comprender mejor las condiciones, situación, intereses y posibilidades de esta población, como insumo para el diseño de programas pertinentes que potencien las acciones que ya están siendo llevadas a cabo. Igualmente, la acción de reconocer se perfila, para los participantes, como un aspecto de aprecio y exaltación que tendría que hacer parte integral de la vida cotidiana de cada docente.

El estudio permite vislumbrar la riqueza y diversidad de las acciones de difusión que los docentes participantes llevan a cabo actualmente. Sin embargo, deja abierta la pregunta de cuán representativos son estos hallazgos en relación con las prácticas del grueso de la población docente, lo cual muestra la importancia de seguir profundizando en estas temáticas.

Al mismo tiempo, permite vislumbrar algunas de las problemáticas más recurrentes que enfrentan los docentes en relación con los procesos de difusión y ofrece pistas de caminos a seguir. En esta línea, hay problemáticas de orden tanto personal -por ejemplo, las limitaciones de tiempo, las diversas capaci- 
dades para la documentación y sistematización, la subutilización de canales digitales-, como estructural -los cambios de rumbo en las políticas públicas o las dificultades existentes para la obtención de información oportuna-. Todas ellas representan retos que pueden tener una respuesta promisoria en un abordaje ecosistémico, que permita identificar oportunidades de apoyo ignoradas hasta el momento.

El mayor desafío, posiblemente, es lograr poner en marcha mecanismos incluyentes -y duraderosque permitan a los docentes una participación activa y visible, aprovechando las oportunidades que transitan en el ecosistema distrital de innovación educativa y les permitan consolidarse como protagonistas de la generación de innovaciones que abran nuevas oportunidades no solo a sus estudiantes, sino a ellos mismos y a las comunidades de las cuales hacen parte.

\section{Agradecimientos y reconocimientos}

Este trabajo es el resultado del esfuerzo conjunto de un grupo de docentes y directivos docentes quienes desarrollan proyectos en innovación educativa en las instituciones educativas oficiales de la ciudad de Bogotá, que con su experiencia y compromiso han aportado en los diferentes escenarios que hacen parte de esta investigación. Igualmente, es fruto del acompañamiento y orientación del equipo técnico del Instituto para la Investigación Educativa y el Desarrollo Pedagógico (IDEP) y de la Dirección de Formación de Docentes e Innovaciones Pedagógicas de la Secretaría de Educación de Bogotá. Para todos ellos un inmenso agradecimiento.

Proyecto financiado por el Instituto para la Investigación Educativa y el Desarrollo Pedagógico (IDEP) de la ciudad de Bogotá, con el acompañamiento de su equipo técnico y de la Dirección de Formación de Docentes e Innovaciones Pedagógicas de la Secretaría de Educación de Bogotá y desarrollado por la Línea de Investigación y Desarrollo en Informática Educativa de la Universidad Eafit, con la participación de profesores del departamento de mercadeo de esta misma institución.

\section{Referencias}

Abdul-Jabbar, M. y Kurshan, B. (2015). Educational ecosystems: A trend in urban educational innovation. Perspectives on Urban Education, 12(1), 7-13. http://proxy-remote.galib.uga.edu/login?url=http://search.ebscohost.

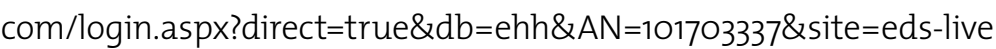

Ahmed, P. K., Shepherd, C. D., Garza Ramos, L. y Ramos Garza, C. (2011). La innovación y su contexto. En Administración de la innovación. Cucienega.

Álvarez Arregui, E., Rodríguez Martín, A. y Ribeiro Gonçalves, F. (2011). Ecosistemas de formación blended-learning para emprender y colaborar en la universidad. Teoría de la Educación. Educación y Cultura en la Sociedad de la Información, 12(4), 7-24. https://www.redalyc.org/pdf/2010/201022649002.pdf

Aronson, J. (1994). A pragmatic view of thematic analysis. The Qualitative Report, 2(1), 1-3. https://nsuworks.nova. edu/tar/vol2/iss1/3

Barrantes, R. (2001). Las innovaciones educativas: escenarios y discursos de una década en Colombia. En M. Henao y J. Castro (comps.), Estados del arte de la investigación en educación y pedagogía (pp. 1-35). Sociedad Colombiana de Pedagogía. 
ISSN 0123-1294 | e-ISSN 2027-5358 | Educ.Educ. Vol. 23. No. 3 | Agosto-octubre de 2020 | pp. 427-443

Universidad de La Sabana | Facultad de Educación

Barraza Macías, A. (2005). Una conceptualización comprehensiva de la innovación educativa. Innovación Educativa, 5(28), 19-31. http://www.redalyc.org/pdf/1794/179421470003.pdf

Carlson, C. R. y Wilmot, W. W. (2006). Innovation: The five disciplines for creating what customers want. Crown Business.

Choque-Larrauri, R. (2009). Ecosistema educativo y fracaso escolar. Revista Iberoaméricana de Educación, 49(4). https://rieoei.org/RIE/article/view/2086

Cortés Salcedo, R. A. (2002). La investigación en el aula: ¿y la innovación pedagógica?. En M. Osorno (ed.), Experiencias docentes, calidad y cambio escolar: investigación e innovación en el aula. IDEP.

Cortés Salcedo, R. A. (2016). Panel sobre investigación e innovación en Bogotá. Simposio IDEP, Bogotá.

Garrido, D., Concepción, M., Rivilla, M., Romero, S. y Mar, V. (2011). La innovación en el aula: referente para el diseño y desarrollo curricular. Perspectiva Educacional, Formación de Profesores, 50(1), 61-86. http://www. perspectivaeducacional.cl/index.php/peducacional/article/view/15

Geertz, C. (2003). La interpretación de las culturas. Basic Books. https://doi.org/10.1017/CBO9781107415324.004

Gómez, I. (2016). Panel sobre investigación e innovación en Bogotá. Simposio IDEP, Bogotá.

Hazen, B. T., Wu, Y., Sankar, C. S. y Jones-Farmer, L. A. (2012). A proposed framework for educational innovation dissemination. Journal of Educational Technology Systems, 4O(3), 301-321. https://doi.org/10.2190/ET.40.3.f

Huberman, A. M. (1973). Cómo se realizan los cambios en la educación: una contribución al estudio de la innovación. Experiencias e innovaciones en educación. Unesco OIE (vol. 4). Unesco. http://unesdoc.unesco.org/ images/0013/001377/137712so.pdf

IDEP (2016). Los centros de innovación en Bogotá. IDEP.

Jackson, D. (2012). What is an innovation ecosystem? National Science Foundation. https://doi.org/10.1017/ CBO9781107415324.004

Lomas, J. (1993). Diffusion, dissemination, and implementation: Who should do what? Annals of the New York Academy of Sciences, 703, 226-237. https://doi.org/10.1111/j.1749-6632.1993.tb26351.x

Markmann, A. (2012). How to create an innovation ecosystem. Harvard Business Review (4 de diciembre), 2-4. https://hbr.org/2012/12/how-to-create-an-innovation-ec?referral=03758\&cm_vc=rr_item_page.top_ right\#comment-section

Melville, N. y Ramirez, R. (2008). Information technology innovation diffusion: an information requirements paradigm. Information Systems Journal, 18(3), 247-273. https://doi.org/10.1111/j.1365-2575.2007.0026o.x

PNUD (2015). Educación de calidad para una ciudad y un país equitativos. PNUD. http://www.co.undp.org/content/dam/colombia/docs/Pobreza/undp-co-educacioncalidad-2015.pdf 
Prahalad, C. K. y Ramaswamy, V. (2003). The new frontier of experience innovation. MIT Sloan Management Review, 44(12), 12-18. http://sloanreview.mit.edu/article/the-new-frontier-of-experience-innovation/

Rogers, E. M. (2003). Diffusion of innovations (5 ed.). Free Press.

Secretaría de Educación de Bogotá (2016). Bogotá reconoce a sus maestras, maestros y directivos docentes líderes de la transformación educativa. [Portal web] http://www.sdp.gov.co/gestion-a-la-inversion/programacion-y-seguimiento-a-la-inversion/proyectos/bogota-reconoce-a-sus-maestras-maestros-y-directivos-docentes-lideres-de-la-transformacion-educativa

Schwandt, T. A. (1994). Constructivist, interpretivist approaches to human inquiry. En Handbook of Qualitative Research (pp. 118-137). Sage.

Shapiro, H., Haahr, J., Bayer, I. y Boekholt, P. (2007). Background paper on innovation and education. European Commission, DG Education \& Culture. https://www.researchgate.net/publication/267955018_Background_Paper_on_Innovation_and_Education

Tobin, D. (2010). What can be expected from educational innovation? Journal of Education Research, 6 (enero), 51-63. https://doi.org/10.13140/2.1.4395.7441

Unesco (2016). Innovación educativa. Herramientas de apoyo para el trabajo docente. Cartolan E.I.R.L. http:// www.unesco.org/lima

Leal Fonseca, D. E., Rojas De Francisco, L. I., Monroy Osorio, J. C., Ortiz Pradilla, T., Delgado Almeida, S. T. (2017). Estrategia de comunicación y divulgación de las acciones que realizan docentes investigadores, innovadores y redes pedagógicas que propicien intercambio de saberes en la ciudad de Bogotá. Informe final, IDEP. https://repositorio.idep.edu.co/bitstream/handle/o01/795/o01idep795.pdf?sequence=1\&isAllowed=y

Zhao, Y., Pugh, K., Sheldon, S. y Byers, J. L. (2002). Conditions for classroom technology innovations. Teachers College Record, 104(3), 482-515. https://www.rtsd.org/cms/lib/PA01000218/Centricity/Domain/96/Conditions\%2ofor\%20Classroom\%20Tech.pdf 